\title{
Grafting Sockets for Fast Container Networking
}

\author{
Ryo Nakamura \\ The University of Tokyo
}

\author{
Yuji Sekiya \\ The University of Tokyo
}

\author{
Hajime Tazaki \\ IIJ Research Laboratory
}

\begin{abstract}
This paper proposes a novel approach for fast container networking that enables applications in containers to utilize host network stacks directly with proper access control. Our approach, called socket-grafting, offers a new socketlayer communication channel by grafting sockets in containers onto sockets in host network stacks. In contrast to recent approaches that make network stacks faster by using high-speed packet I/O techniques, socket-grafting makes container networking faster by bypassing a container's network stack and virtual interfaces. As a concrete implementation of socket-grafting, we designed AF_GRAFT, which is a new address family for the socket layer. AF_GRAFT performs interconnection between the two socket layers in the container and host network stacks. In this paper, we describe socket-grafting, AF_GRAFT design, and its implementation on Linux. Our evaluation results demonstrate that the proposed method doubled throughput and reduced latency by $23 \%$ compared with traditional NAT-based container networking, and improved the network performance of containerized HTTP servers and message queues.
\end{abstract}

\section{CCS CONCEPTS}

- Networks $\rightarrow$ Cloud computing; $\bullet$ Software and its engineering $\rightarrow$ Communications management;

\section{KEYWORDS}

container networking, network stack, socket API

\section{ACM Reference Format:}

Ryo Nakamura, Yuji Sekiya, and Hajime Tazaki. 2018. Grafting Sockets for Fast Container Networking. In ANCS '18: Symposium on Architectures for Networking and Communications Systems, fuly 23-24, 2018, Ithaca, NY, USA. ACM, New York, NY, USA, 13 pages. https://doi.org/10.1145/3230718.3230723

Permission to make digital or hard copies of all or part of this work for personal or classroom use is granted without fee provided that copies are not made or distributed for profit or commercial advantage and that copies bear this notice and the full citation on the first page. Copyrights for components of this work owned by others than ACM must be honored. Abstracting with credit is permitted. To copy otherwise, or republish, to post on servers or to redistribute to lists, requires prior specific permission and/or a fee. Request permissions from permissions@acm.org.

ANCS '18, fuly 23-24, 2018, Ithaca, NY, USA

(c) 2018 Association for Computing Machinery. ACM ISBN 978-1-4503-5902-3/18/07 ..\$15.00 https://doi.org/10.1145/3230718.3230723

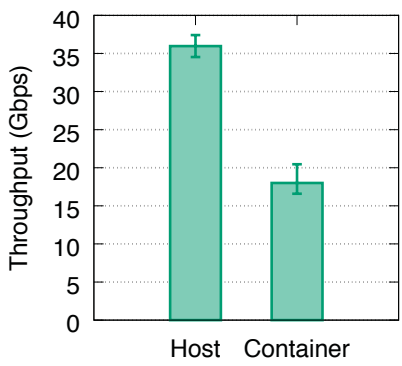

(a) Throughput

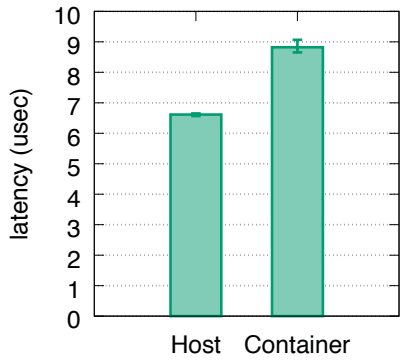

(b) Latency
Figure 1: Performance comparison of a native host and a container with the docker default networking.

\section{INTRODUCTION}

Container technologies have enabled new computing environments, such as microservice architecture [13,22] and serverless computing $[4,31]$. In such design principles, a monolithic system is decomposed into small services. A single service of the system is implemented in a container, and it is deployed into on-premise or off-premise container clusters. The container's portability and lightweight virtualization enable these environments where many containers run concurrently at the same time.

A container image is a portable, version-controllable package of an application execution environment. All the parts to run an application, e.g., libraries, configurations, and codes, are maintained in a container image. People can make their images, and run the images on any infrastructure that support the same container engine. A container that a container image runs is a separated space in a host operating system (OS). In contrast to virtual machines, container-based virtualization is the separation of instances of OS components, such as network, file system, process ID, etc. Therefore, containers generally achieve better performance than virtual machines because containers do not involve device emulation overhead and I/O across virtualization layers [17, 25].

Although container-based virtualization is lightweight compared with virtual machines, both the network throughput and latency of containers are still worse than native bare-metal hosts. Figure 1 shows the results of our preliminary experiment. In this experiment, we prepared two Linux machines connected to each other via a 40 Gbps link, and measured throughput and message round-trip latency from a docker container or the machine to the other machine using iperf3 and sockperf [34] (details of the setup are described 
in Section 5). As shown in Figure $1 \mathrm{a}$ and $1 \mathrm{~b}$, the container degraded throughput in half and increased latency by $23 \%$ compared with the native host. A container is a separated namespace on a host OS. Thus, some network functionalities are required to connect the separated container's network stack to others and external networks. In typical default container networking [3, 9, 24], host network stacks perform NAT routers for containers, and virtual Ethernet interfaces perform packet I/O channels between containers and the host. This additional network stack processing and communication using virtual interfaces cause the network performance degradation compared with native hosts.

To overcome the degradation of throughput and latency in current container networking, we propose a new approach, called socket-grafting. Socket-grafting grafts sockets created in containers onto sockets created in host network stacks. This is a new communication channel design between container and host spaces. As an implementation of socketgrafting, we designed and developed AF_GRAFT, which is a new address family for grafting sockets. Applications in containers create AF_GRAFT sockets, and then the bind() system call grafts the sockets onto the specified sockets in host network stacks across the separation of container and host spaces. Besides, an access control mechanism through the bind() semantics prevents malicious or unintended uses of host namespaces. By using this method, container network stacks are bypassed, and virtual interfaces are removed.

We implemented AF_GRAFT in Linux kernel, and a library, which dynamically converts existent application binaries into AF_GRAFT-capable by using a technique for overriding functions. With these implementations, we evaluated network performance improvement on basic network benchmarks and two typical container applications: throughput, latency, HTTP server (NGINX) and message queuing (ZeroMQ). The evaluation results show that our method can eliminate the network performance degradation due to current container networking. AF_GRAFT approximately doubles throughput and reduces latency by $23 \%$ with a single TCP flow compared with traditional NAT-based container networking. Moreover, HTTP and message queue benchmarks demonstrate that AF_GRAFT can improve the network performance of typical containerized applications.

The contributions of this paper include the following:

- A new approach for bypassing container network stacks to overcome the network performance degradation of containers.

- The design and implementation of AF_GRAFT, which is an implementation of socket-grafting.

\section{BACKGROUND}

A container is a separated namespace in a host OS. Therefore, a container's network stack is also separated from a host network stack, and it should somehow connect to other containers, the host network stack, and external networks. This is the beginning of container networking. A conventional approach for connecting different network entities is to use network adapters and links, and current container networking follows this approach. Container and host network stacks are connected across virtual interfaces (veth interfaces in Linux), and the host network stack performs a packet forwarder such as bridge, router, NAT, and sometimes tunneling. For this additional network processing, the throughput and latency of containers degrade compared with native hosts. This section investigates overheads of container networking and summarizes recent approaches that attempt to overcome the degradation. We also describe the motivation for exploiting the socket layer as a new communication channel for containers.

\subsection{Overhead of Container Networking}

The requirements on networking for containers in a host OS are the separation of namespaces (i.e., addresses, protocols, and ports) between containers and the host, and a communication channel between containers and external networks. Linux achieves this separation by creating individual network stack instances for containers. Additionally, virtual interfaces connect the separated network stack instances. Figure 2a depicts the network stack architecture of this traditional container networking.

The degradation of throughput and latency demonstrated in Figure 1 arises from the long data path of the container network stack architecture. In a native host, packets pass through a host network stack and a physical NIC. In a container, packets pass through a container's network stack, a virtual NIC, a bridge interface on the host network stack, the host network stack for IP routing and NAT, and the physical NIC. Thus, time to transmit a packet increases, and throughput and latency degrade as a result.

To demonstrate this long data path, we investigated the UDP transmission path on Linux using ftrace, a function tracer for Linux. Figure 3a shows the depth of all called functions from udp_sendmsg( ), which is an entry function of the UDP transmission path, as the start point. The called functions consequent on udp_sendmsg() will reach a function to write packets to the physical NIC at the end of the long data path. Therefore, over half of the called functions in the container are deeper than the deepest function of the data path in the native host. Indeed, the required time to transmit a packet increases. Figure $3 \mathrm{~b}$ shows the average and standard deviation of elapsed times in udp_sendmsg() while sending 


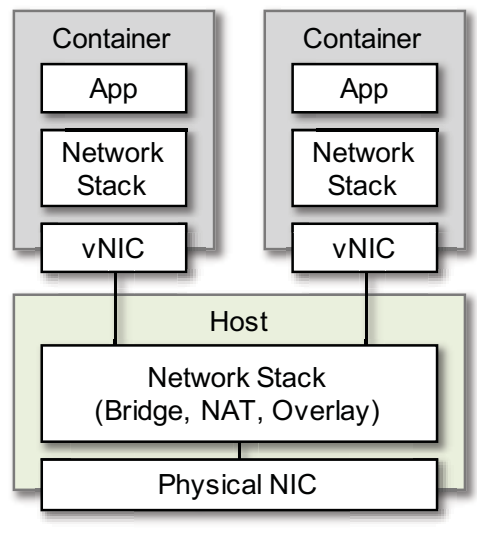

(a) Traditional Container Networking.

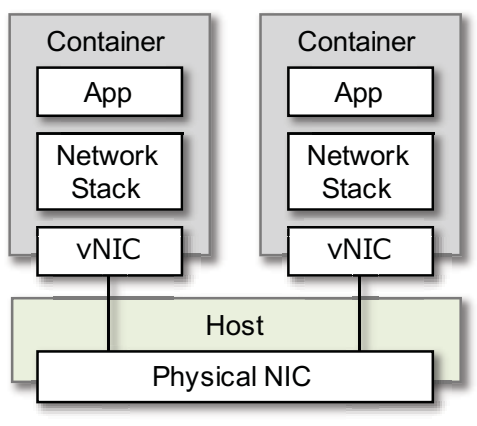

(b) Interface Virtualization.

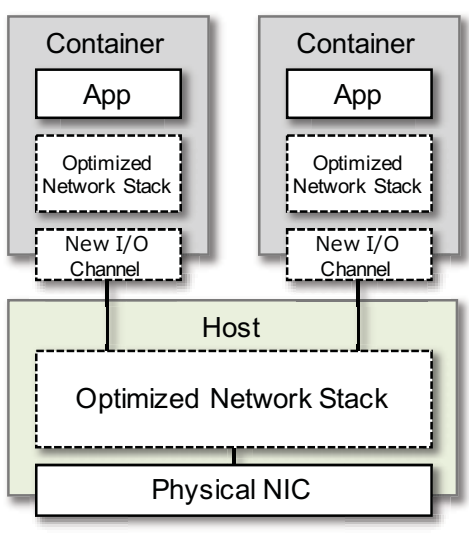

(c) Optimized Network Stack.

Figure 2: Network stack architectures for container networking. Interface virtualization bypasses the host network stack, and the optimized network stacks reinvent network stacks in containers and the host.

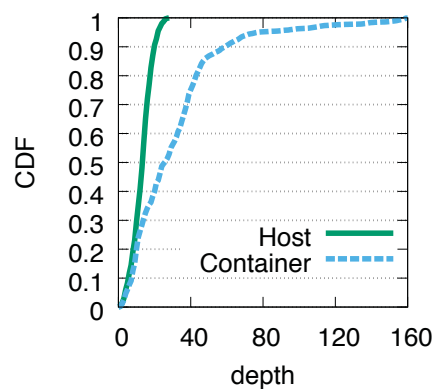

(a) Depth of called functions from udp_sendmsg().

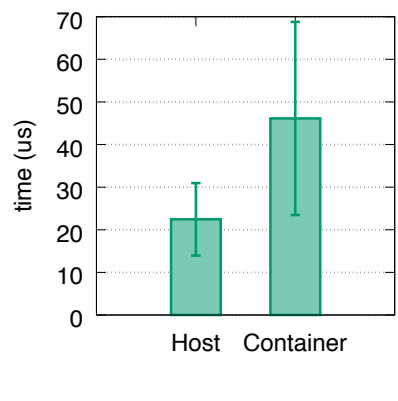

(b) Elapsed time in udp_sendmsg().

Figure 3: Demonstration of the long data path.

UDP packets using iperf3. The required time to transmit a packet is approximately doubled in the container because of the long data path involving two network stacks.

\subsection{State-of-the-Art Container Networking}

There are two types of approaches for overcoming the network performance degradation due to the container network architecture: (1) interface virtualization and (2) optimizing network stacks. The first approach is to connect containers with outer networks bypassing host network stacks. The second approach makes network stacks faster using high-speed packet I/O techniques. Figure $2 \mathrm{~b}$ and Figure 2c illustrate the network stack architectures of the two approaches.

For reducing network stack processing, one of the simplest ways is to attach physical interfaces to containers directly; however, this is a waste of interfaces. Interface virtualization techniques, such as macvlan and SR-IOV, enable multiple virtual interfaces hiring a single physical interface. Containers

having these virtual interfaces can bypass the host network stack as illustrated in Figure 2b. Previous studies reported that the macvlan interface outperforms the throughput of default container networking $[14,38]$. However, the interface virtualization approach has a drawback: containers directly connect to outer networks, so that the underlying network infrastructure and its management are complicated. In the case that containers are behind host network stacks performing NAT routers, the container networks and outer networks are decoupled, and operators can assign arbitrary IP addresses to containers. However, if containers directly connect to the outer network of the host, address and routing management for containers is required in the outer network. Moreover, some firewalls should be also deployed in the outer network.

Another approach is to reinvent the entire or a part of network stacks using high-speed packet I/O techniques such as the Data Plane Development Kit (DPDK) [2] or netmap [29]. This is a feasible way for fast container networking even though it requires considerable effort to reimplement a wide range of networking features. Nevertheless, regardless of how fast the network stack is, the network stack and its protocol processing cost do not disappear. It means that the protocol processing cost will be the next bottleneck. For example, StackMap [35] reported that TCP/IP protocol processing is relatively cheap because the socket API and packet I/O are currently dominant. In Arrakis OS [27], which has a very optimized I/O mechanism, network stack processing occupies $100 \%$ of the processing cost on a simple UDP echo server. These reports suggest that if network stacks including packet I/O are sufficiently fast in the future, the long data path through the container and host network stacks will be a more significant bottleneck in comparison with native hosts unless the container network stack architecture is changed. 


\subsection{Motivation}

The previous section described the two state-of-the-art approaches for fast container networking and their drawbacks: interface virtualization techniques bypassing host network stacks complicate outer network management, and optimizing network stacks will involve significant overhead due to the long data path unless the container network stack architecture is changed.

As a third approach, we advocate bypassing container network stacks. A container is just an application execution environment. An application, which is responsible for a small service comprising a system, runs on a container. If this application focuses on only data above the socket layer, then the container's network stack would be unnecessary.

For example, let us consider a simple HTTP server. An HTTP server exchanges the same data regardless whether its network stack is the host's or the container's. An HTTP server running on a docker container is usually exposed to the Internet using a static NAT entry in the host network stack. Clients connect to the HTTP server using a global IP address that the host network stack has, but network stack processing occurs also in the container's network stack. In this case, if the HTTP server runs on top of the host network stack, the container's network stack processing and data delivery across virtual interfaces are removed. Moreover, communications from the viewpoint of the clients and the HTTP server remain the same: the clients connect to host's global IP address, and the HTTP server sends and receives the same data regardless of the existence of the NAT.

Running an application on a host network stack while maintaining separation for other components achieves this optimization; however, it is not feasible from a security perspective. The docker supports sharing the host network stack with containers, e.g., docker run --net=host. However, in the case of container-based clouds where container users and host operators are different, the container users can exploit unauthorized network resources, e.g., physical NICs, IP addresses and port numbers, which can lead to malicious uses. Therefore, network stack separation is still a necessary key component for container-based virtualization.

Therefore, we advocate that the container network stacks can be bypassed for throughput and latency if applications running on the containers are insensitive to network stacks. At the same time, network stack separation should be retained for security reasons. We believe this hypothesis can apply to a wide range of containerized applications: web servers, database servers, message queues for interaction between services, etc. Such applications are interested in data coming from connections through sockets and are not interested in how packets are delivered.

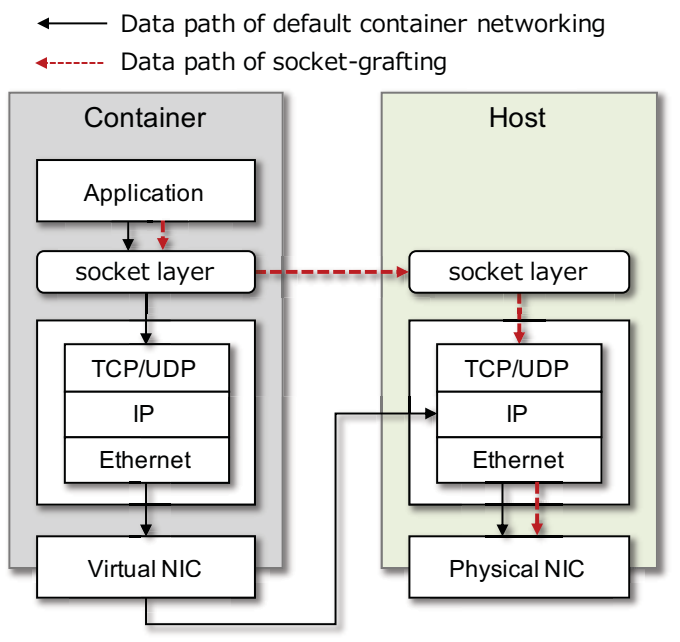

Figure 4: The data path of socket-grafting. Socketgrafting creates a new communication channel between the two socket layers in the container and the host.

\section{SOCKET-GRAFTING}

For feasibly bypassing container network stacks, we propose a new data communication channel design between container and host network stacks. Our approach, called socket-grafting, provides interconnection between sockets opened in container network stacks and sockets opened in host network stacks. Namely, it is a socket-layer communication channel instead of a virtual interface.

Figure 4 illustrates the data path of socket-grafting. In contrast to the long data path of the current container network architecture, it involves a single network stack. An application in a container opens a socket, and then this socket is grafted onto a socket opened at a host network stack. On the transmit side, data written to the socket in the container is directly delivered to the socket in the host network stack. The host network stack processes network protocols and transmits the packets to the destination. On the receive side, applications in containers read received data from the host network stack through the graft. Unlike socket pairs in which data written to a socket is read from the other socket, data written to a socket in a container is delivered and also written to a socket in the host.

One advantage of socket-grafting is network performance improvement by bypassing the container network stacks. As depicted in Figure 4, the data path of socket-grafting does not involve the container's network stack and packet I/O through virtual interfaces. Thus, socket-grafting can overcome the network performance degradation due to the long data path of the container network architecture. Another advantage of socket-grafting is that it is a new communication 
channel design in the socket layer; therefore, it does not depend on the implementations of network stacks. The socketgrafting approach can be applied to existent full-featured network stacks of general purpose OSs and optimized network stacks implemented from scratch. If current network stacks are replaced with high-speed network stacks in the future, socket-grafting will be able to help the network performance of containers by reducing the processing cost of a network stack.

\subsection{AF_GRAFT Design}

We developed socket-grafting as a new address family, called AF_GRAFT.AF_GRAFT sockets can be grafted onto other address family sockets, i.e., AF_INET, AF_INET6, and AF_UNIX, across the separation of network stacks. Socket operationsconnect (), accept (), write (), read(), etc.-to AF_GRAFT sockets are transferred to actual sockets opened in host network stacks (we call these host sockets). In addition, AF_GRAFT provides an access control mechanism to prevent malicious or unintended uses of the host namespace, i.e., addresses and port numbers.

To cooperate with different types of sockets, we designed a mechanism to map AF_GRAFT sockets onto host sockets. For this mechanism, we utilize the bind() system call and endpoints of sockets. Endpoints are the same as names in the bind() semantics. For example, an endpoint is a pair of an IP address and a port number for AF_INET and AF_INET6, or a file system path name for AF_UNIX. As well as such address families, AF_GRAFT has its own type of endpoints in which sockets can be assigned similarly. Figure 5 shows an example where three containers utilize AF_GRAFT on a host. We call the endpoints for AF_GRAFT graft endpoints. A graft endpoint, which is an arbitrary string, is associated with an actual endpoint in the host network stack (host endpoints). In this example, Container 1 has a graft endpoint ep-http that is associated with the host endpoint 10.0.0.1 and port 80. When an application in Container 1 creates a socket with AF_GRAFT and assigns the socket to ep-http by the bind() system call, the socket is grafted onto the AF_INET socket assigned to 10.0.0.1:80 on the host network stack.

This mechanism simultaneously performs access control to limit host endpoints that individual containers can use for their host sockets. AF_GRAFT manages the namespace of graft endpoints per container. Thus, containers can use only specific host endpoints exposed to the containers as graft endpoints. Table 1 shows which containers have which graft endpoints in the example shown in Figure 5. Container 1 can use only 10.0.0.1:80, and Container 2 can use only 10.0.0.1:8080 and [2001:db8::beef]:8080. This limitation prevents misuse of the host namespace.

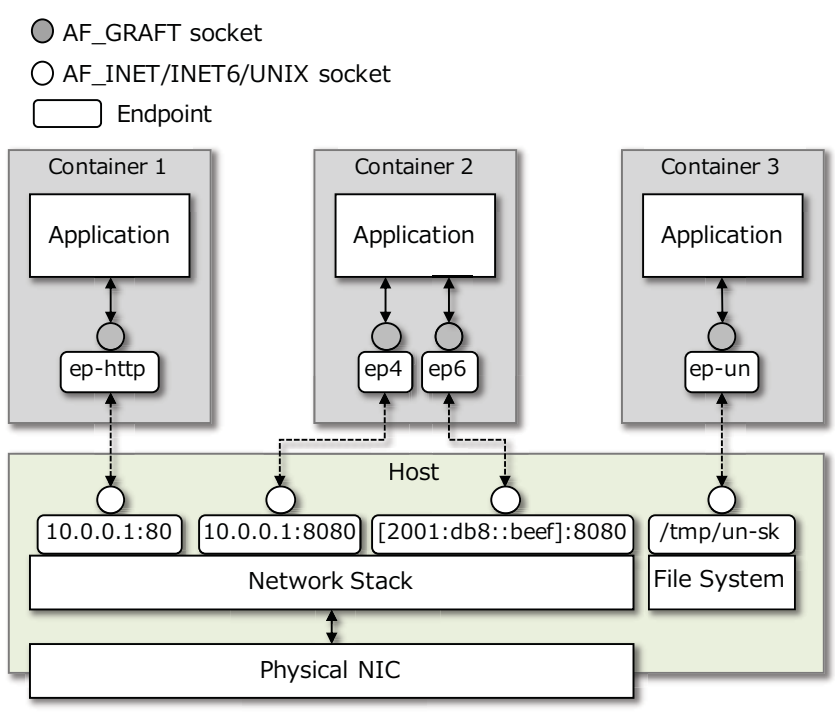

Figure 5: An example of the use of AF_GRAFT sockets. Three individual containers utilize limited endpoints on the host network stack across the AF_GRAFT socket-layer connection.

Table 1: The example of graft endpoints and associated actual endpoints in the host shown in Figure 5. AF_GRAFT manages these tables in each container.

\begin{tabular}{r|r|l}
\hline Container & Graft endpoints & Host endpoints \\
\hline Container 1 & ep-http & $10.0 .0 .1: 80$ \\
\hline Container 2 & ep4 & $10.0 .0 .1: 8080$ \\
& ep6 & [2001:db8::beef]:8080 \\
\hline Container 3 & ep-un & /tmp/un-sk \\
\hline
\end{tabular}

A life cycle of an AF_GRAFT socket in a container is described below: An application creates an AF_GRAFT socket with proper protocol and type. At this time, the corresponding host socket is not created, because the address family of the host socket is indefinite until its host endpoint is determined by bind(). When the application calls bind() to assign the socket to a graft endpoint, AF_GRAFT then creates an actual host socket on the host endpoint associated with the graft endpoint. This host socket has the identical protocol and type as the AF_GRAFT socket in the container. After this bind() process has completed successfully, AF_GRAFT starts to transfer all socket operations to the AF_GRAFT socket in the container to the host socket. For example, the application calls listen () to the AF_GRAFT socket, and the host socket starts to listen. The application closes the socket, and the host socket is also closed. 


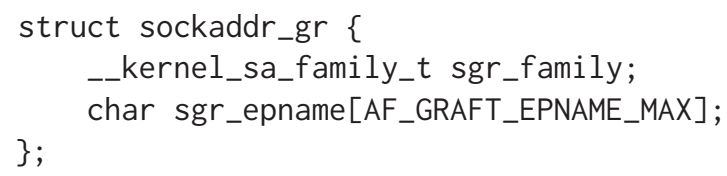

Figure 6: The sockaddr_gr structure in Linux. Note that _-kernel_sa_family_t is changed to uint8_t length and sa_family_t in BSD OSs.

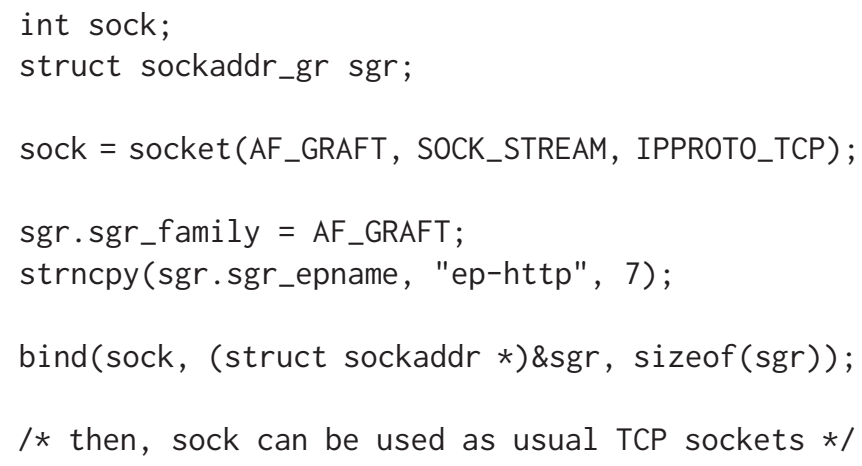

Figure 7: How to create and bind AF_GRAFT sockets.

\subsection{AF_GRAFT Socket API}

The AF_GRAFT socket API follows the traditional socket API: create a socket using socket () and assign the socket to a graft endpoint using bind (). For adapting graft endpoints for bind(), we introduce a new socket address structure for the AF_GRAFT address family. Figure 6 shows this sockaddr_gr structure. It contains the address family identifier and a string buffer for a graft endpoint name. Applications call bind() with sockaddr_gr containing graft endpoint names to which the sockets are assigned.

Figure 7 shows an example code to use an AF_GRAFT socket. Except for a few bind()-related code lines, it is identical to the use of other address family sockets. After bind() returns, the socket can be used as usual TCP sockets: the application can call listen(), accept(), read(), write(), and so on.

\subsection{Outbound Connections}

Previous sections focused on inbound connections toward containers. Naturally, the AF_GRAFT mechanism can work for outbound connections from containers to external hosts. For AF_INET and AF_INET6 outbound connections, anyport graft endpoints can be used. Any-port endpoints do not specify port numbers, unlike the examples shown in Figure 5. When an application in a container assigns an AF_GRAFT socket to an any-port endpoint, the corresponding host socket is created and assigned to the IP address and a randomly selected port number. In other words, the any-port endpoints create typical client sockets having random source port numbers. After grafting, the application can call connect() for the AF_GRAFT socket, and then the host socket starts connect() to the destination. Similarly, any-path endpoints, which do not specify paths of the UNIX domain socket, can work for connect() on sockets grafted onto UNIX domain sockets.

\subsection{Integration with Container Platforms}

Endpoints are network resources shared among multiple containers and host network stacks. Thus, containers should not configure and utilize endpoints arbitrarily by themselves. Instead, only container platforms should configure. Indeed, today's container runtimes and orchestration platforms have mechanisms for configuring container network stacks, e.g., IP addresses and routing tables. Such mechanisms will be able to manage AF_GRAFT.

For exposing applications running on containers to external clients, typical container runtimes have execute options for configuring static NAT entries, e.g., docker run -p $8080: 80$. IP addresses and ports of host network stacks are mapped to specified containers when the containers start. AF_GRAFT can substitute for these static NAT entries. When pairs of host and graft endpoints are given as options, container runtimes can configure the graft endpoints in containers. Like the NAT maps IP addresses and ports of containers to those of hosts, AF_GRAFT connects sockets opened in containers to sockets opened in host network stacks.

In container platforms such as Kubernetes [7], it is slightly different because NAT performs decoupling of service and multiple containers. Kubernetes manages a couple of containers as a Pod where the containers share a single, separated network stack. Every Pod has a unique private IP address on its network stack, and a Service abstracts multiple Pods under an IP address (cluster IP). Packets to the cluster IP are distributed among the Pods using destination NAT for load balancing. The Service abstraction with NAT conceals life and death of containers for scaling up or down. In this network architecture of Kubernetes, AF_GRAFT enables the bypassing the Pod's network stack. Instead of the private IP addresses in the Pods, the host network stack should have additional IP addresses. Applications in the containers open sockets on the additional IP addresses through AF_GRAFT, and packets to the cluster IP is distributed among the addresses by destination NAT. Implementing AF_GRAFT as a Container Network Interface (CNI) [6] plugin would achieve this integration.

\subsection{Implementation}

We implemented AF_GRAFT as a single kernel module for Linux. When this module is loaded, AF_GRAFT is registered 
as an address family in the socket layer. This module does not require any patches to the kernel codes. However, it currently has to overwrite an existing address family number. The address family numbers are strictly defined and dynamically adding a new number is prohibited in the Linux kernel.

Data and operation delivery between AF_GRAFT sockets and host sockets is function call. There are no messaging, buffering, or queuing mechanisms. Therefore, its overhead is minimal. For example, the implementation of sendmsg() of AF_GRAFT sockets simply calls the implementation of sendmsg() of the corresponding host socket. Similarly, recvmsg() of AF_GRAFT calls recvmsg() of host sockets. Other socket operations, i.e., accept, listen, connect, setsockopt, etc., are also implemented in the same manner. Only bind() works differently as described in Section 3.1. bind() creates a host socket and then calls bind() for the host socket with the corresponding host endpoint.

We implemented a few socket options for practical uses. For distinguishing AF_GRAFT socket options from others in the kernel side, we introduced IPPROTO_GRAFT as a new socket level (the second argument of setsockopt()). The first option is transparent name option. getsockname() returns the endpoint name of the specified socket; however, the AF_GRAFT socket has two endpoints: graft and host. The transparent name option switches which endpoint getsockname() returns. Note that getpeername() returns the endpoint of the peer connected to the socket when this option is enabled. The second option is the delayed execution of setsockopt. When this option is set on, all setsockopt () operations excluding IPPROTO_GRAFT are queued. The queued operations are executed when bind() for the AF_GRAFT socket is called and the host socket is created. The delayed execution of setsockopt enables applications to call setsockopt() before actual host sockets are created. This mechanism helps to convert existing applications to AF_GRAFT-capable using system call interception techniques described in Section 4.

To configure graft endpoints, we enhanced iproute2 [8], which is the best-known network configuration tool in Linux. As with the familiar usage of the ip command such as routing table entry insertion or deletion, users and container platforms can add and delete graft endpoints like commands shown below.

\$ ip graft add ep-http type ipv4 addr 10.0.0.1 port 80 \$ ip graft add ep-out type ipv4 addr 10.0.0.2 port any $\$$ ip graft del ep-un

The source codes described in this paper are available [10].

\section{SYSTEM CALL INTERCEPTION FOR NON-AF_GRAFT APPLICATIONS}

AF_GRAFT is a new address family. Thus, existing applications supporting only AF_INET and AF_INET6 sockets do not work with AF_GRAFT. Even if modifying their codes to support AF_GRAFT does not require significant effort because of the familiar socket API, it is still difficult to deploy them in practical environments. Maintaining out-of-tree codes is usually harder than using mainstream codes and packages as is.

For those non-AF_GRAFT-capable applications, we utilize system call interception techniques for converting address family-dependent socket operations into AF_GRAFT-capable ones. ptrace is a technique for system call interception. It enables us to attach a process (the tracer) to another process (the tracee), and the tracer intercepts system calls from the tracee. LD_PRELOAD also allows dynamic function override by third-party shared libraries [28]. By using these techniques, system call behaviors can be overridden dynamically without modifications to application codes [18, 26]. We implemented a shared library, called libgraft-hijack, that dynamically overrides socket-related functions to convert other address family sockets into AF_GRAFT sockets. By using this library with LD_PRELOAD, existing applications can obtain the benefit of the use of AF_GRAFT without modifications.

Note that this technique is not always applicable to all applications. Functions that can be overridden must be called via the procedure linkage table. Thus, it cannot override functions in statically linked libraries and hidden symbols. Fortunately, usual applications are applicable because they invoke socket-related functions via dynamically linked GNU C Library (glibc). Another exception is the Go Programming Language. Go implements system calls without the glibc; therefore, this technique does not work with applications written in Go.

\section{1 getaddrinfo()}

We developed two methods for the conversion to AF_GRAFT. The first is to override getaddrinfo(). The getaddrinfo() function converts host names and service names (port numbers) into proper sockaddr structures. Server applications can use this function to obtain sockaddr structures to which the applications assign sockets. The modified getaddrinfo() by our library returns a sockaddr_gr structure if the queried host name matches a graft endpoint name and the queried address family is AF_UNSPEC or AF_GRAFT. getaddrinfo() was carefully designed to make application codes independent from specific address families and protocols [21]. Therefore, if a server application was written in such a manner, the application could cope with AF_GRAFT sockets transparently. However, unfortunately, this is not the case in practical applications. Almost all applications strictly check address families or assume that the returned families are IPv4 or IPv6. Thus, this method does not work in most cases. 


\section{2 socket(), bind() and connect()}

The second method converts socket () and bind() for server applications, and connect () for client applications. In contrast to the getaddrinfo() case, this method can deal with address family-dependent applications.

Figure 8 shows the function call flow for converting an AF_INET server socket into AF_GRAFT. The key points are (1) overriding socket () to return an AF_GRAFT socket, and (2) overriding bind() to convert original sockaddr structures to proper sockaddr_gr structures. First, an application in a container calls socket (), and the library creates and returns an AF_GRAFT socket. This socket is created with the same type and protocol. Moreover, the library enables the delayed setsockopt execution option on this socket. At this point, the host socket has not been created yet. Therefore, if the application calls setsockopt () excluding AF_GRAFT socket options on this socket, it fails. The delayed execution of setsockopt prevents this failure. The most typical socket option that fails is the SO_REUSEADDR option. It is called after socket () and before bind().

After the socket descriptor returns to the application, the application calls bind() with a sockaddr structure, e.g., sockaddr_in in Figure 8. The library converts this sockaddr structure to the proper sockaddr_gr structure. In the current libgraft-hijack implementation, the mapping configuration that specifies which sockaddr should convert to which sockaddr_gr is given by an environment variable ${ }^{1}$. When the library calls bind() with the sockaddr_gr, the AF_GRAFT kernel module creates the host socket and starts grafting, and the bind() returns on the application context. The delayed socket options are executed after the host socket is created and before bind() for the host socket is called. Lastly, the application transparently obtains the server socket for listen(), accept(), and so on.

Converting client sockets to AF_GRAFT is quite simple. It is done by overriding connect() and executing bind() before connect(). When applications do not need to specify source addresses and ports of sockets, they call connect() without bind (). However, AF_GRAFT requires calling bind() with sockaddr_gr to determine actual host sockets. First, the library overrides socket () as well as the server sockets. Next, when the application calls connect(), the library overrides and calls bind() with sockaddr_gr of the given any-port graft endpoint ${ }^{2}$ before connect(). As a result, the AF_GRAFT socket starts to work as a conventional client socket assigned to the specified host IP address and the randomly selected port. Note that sendto() and sendmsg() are also overwritten in the same manner because they do not need to call bind() and connect() in advance.

\footnotetext{
${ }^{1}$ GRAFT_CONV_PAIRS $=" 0 \cdot 0 \cdot 0 \cdot 0: 80=\mathrm{ep}-\mathrm{http} "$ for instance.

${ }^{2}$ As with the mapping, it is configured by an environment variable.
}

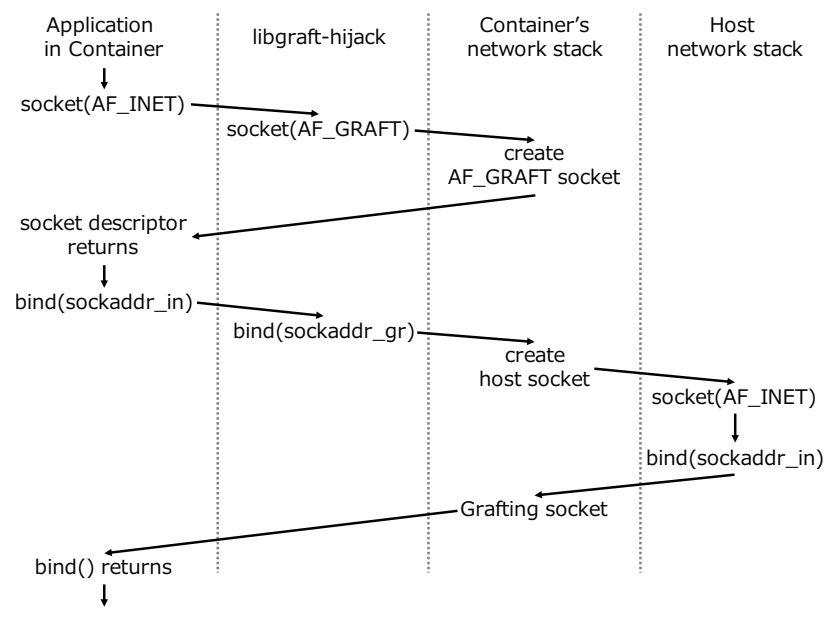

Figure 8: The sequence of overriding to convert the AF_INET socket into the AF_GRAFT socket.

\section{EVALUATION}

In this section, we evaluate the network performance improvement by AF_GRAFT. When using AF_GRAFT, the components that packets pass through are the same as the native hosts: the socket layer, a network stack, and a physical NIC. Therefore, network performance, e.g., throughput and latency, of the applications in containers with AF_GRAFT will overcome the degradation due to the long data path and will be comparable with that of the native hosts.

To demonstrate this improvement, we conducted three experiments: (1) throughput and latency measurements for clarifying baseline performance, (2) an HTTP server benchmark, and (3) a message queue benchmark. Both the HTTP and message queue are popular applications running on containers. In addition to the regular role as web content servers, HTTP has been widely adopted for communication between applications via such REST API. Therefore, network performance improvement on HTTP servers in containers has the potential to contribute to containerized microservices. Message queuing is also used in such decomposed systems for message passing between services.

For the experiments, we prepared two machines with Linux kernel 4.4.0, Intel Core i7-3770K 3.5 GHz CPU, 32 GB of DDR3-1600 RAM, and Mellanox ConnectX-4 LX 40 Gbps NICs. The two machines were directly connected to each other with a $40 \mathrm{Gbps}$ link. In most of our experiments, an application on a docker container in the machine sent or received packets, and the other measured its performance. When using the docker container, its network was the docker default NAT network (docker 0 ). Additionally, the MTU of all the NICs was 1500 bytes. This environment is the same as the preliminary experiments described in Section 1 and 2.1. 


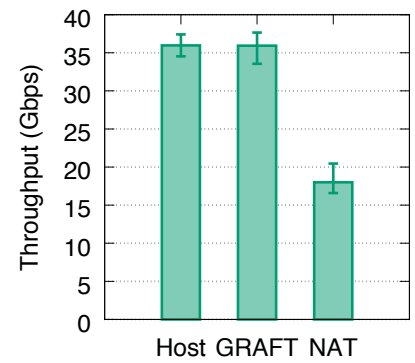

(a) Transmit throughput

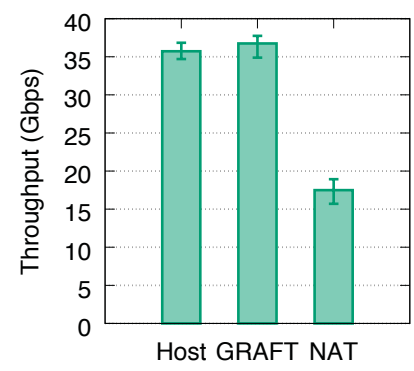

(b) Receive throughput
Figure 9: Throughput with a single TCP flow.

For the baseline benchmark, we used iperf3 for the throughput test and sockperf for the latency test as well as in the preliminary experiments. For the HTTP server and message queue benchmarks, we used NGINX 1.10.3 and siege 3.0.8, which is an HTTP benchmark tool, and ZeroMQ 4.2.2 and its built-in benchmark application. The container runtime was docker 17.12.0. To support AF_GRAFT on these applications, we used the second conversion method described in Section 4.2.

\subsection{Throughput}

In the first experiment, an iperf3 process in the container transmitted or received IPv4 TCP single flow test traffic to or from an iperf3 process running on the other machine. Figure 9 shows the result of this experiment. In the key, Host, GRAFT, and NAT indicate the result of native host-tohost communication, the results when the container used AF_GRAFT, or the default docker NAT network, respectively. Note that all the results in this section show the maximum, minimum, and average of 20 runs on each permutation.

As shown in Figure 9, AF_GRAFT overcomes the performance degradation due to the NAT-based container networking on both the transmit and receive sides. The throughput of docker 0 NAT was approximately $18 \mathrm{Gbps}$. On the other hand, the container using AF_GRAFT achieved 35 Gbps as well as the native host. This result demonstrates that AF_GRAFT successfully eliminates overheads from virtual interfaces and the additional network stack processing in the container. Moreover, the overhead of data and operation delivery between the two sockets is negligible since AF_GRAFT implements it as function call.

Next, we measured the throughput between two docker containers in the same host. Figure 10 shows this result. The AF_GRAFT throughput is $30 \%$ or $75 \%$ faster than the bridging by docker $\theta$ in TCP or UDP, respectively. The communication between the two containers using AF_GRAFT is the same as inter-process communication via the loopback interface. The processes in the containers connect to each other using the

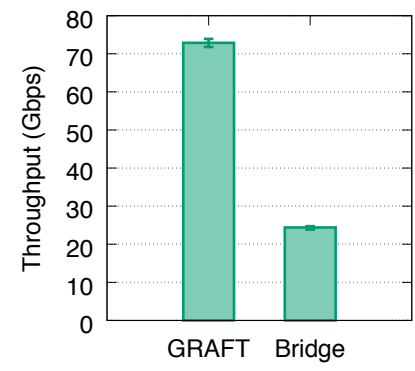

(a) TCP

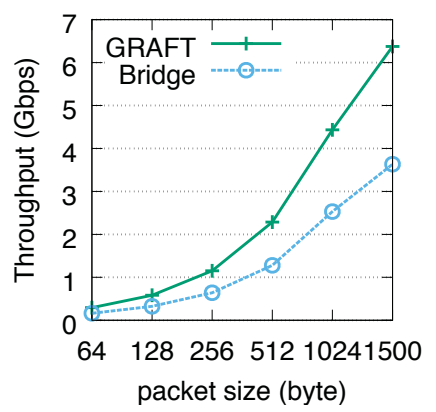

(b) UDP
Figure 10: Throughput between the two containers on the same host.

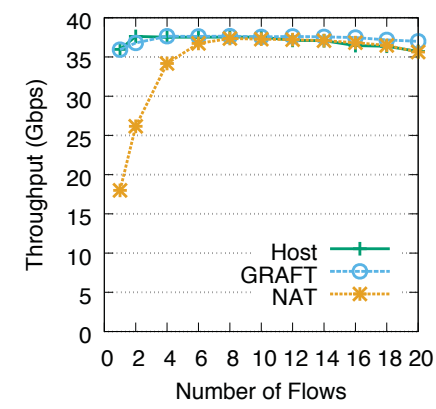

(a) Transmit throughput

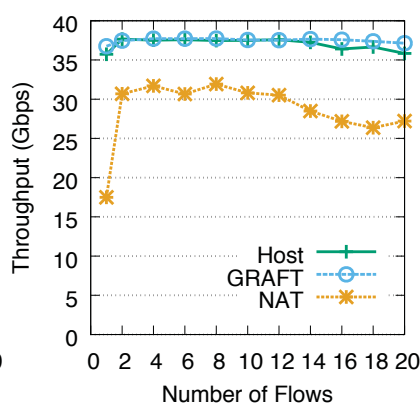

(b) Receive throughput
Figure 11: Throughput with multiple TCP flows.

host sockets opened at the host network stack. Therefore, the nonexistence of virtual interfaces, i.e., the bridge and the veth interfaces, produces this result. Note that the MTU size of the loopback interface was 65536, which was significant for the TCP throughput. However, TCP segmentation offload on the docker $\theta$ bridge was enabled, so that there were no differences caused by the MTU sizes.

In addition to the single flow tests, we measured interhost throughput with the test traffic composed of multiple flows. Figure 11 shows the results. As well as the single flow tests, AF_GRAFT achieves throughput comparable with the native hosts. On the transmit side, the NAT-based container network also reaches $35 \mathrm{Gbps}$ with six flows. In contrast, on the receive side, its throughput is approximately $30 \mathrm{Gbps}$ regardless of the number of flows. In addition to the overhead from the long data path, this is because Linux kernel bridges (docker 0 ) do not support large receive offload [15] ${ }^{3}$. Therefore, its receive throughput is poorer than the native hosts and AF_GRAFT, which can directly utilize the host network stack and the physical NIC.

\footnotetext{
${ }^{3}$ https://github.com/moby/moby/issues/32023
} 


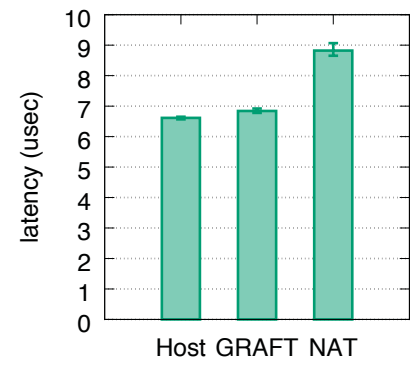

(a) TCP

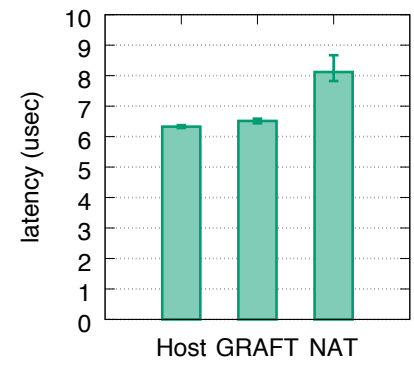

(b) UDP
Figure 12: Message round-trip latency.

\subsection{Latency}

For the second baseline benchmark, we measured latency using sockperf. Similar to the preliminary experiment, we measured message round-trip latency between a container and a host: the container in the machine sends a message, and the other machine returns the message. Note that the message size was 14 bytes, which is the minimum size of sockperf. Figure 12 shows the results of both TCP and UDP test cases. As well as the throughput test results, AF_GRAFT overcomes the degradation from the latency perspective. Latency is one of the most critical metrics for service responsiveness and user experience, especially microservice architecture [11]. Therefore, AF_GRAFT, which reduces latency by about $23 \%$ compared with the NAT-based container network, will help the responsiveness of such services in the containers.

\subsection{HTTP Server Benchmark}

So far, we have measured the primitive performance: throughput and latency. It is therefore interesting to measure improvement in containerized applications. As a target application, we conducted a simple HTTP server benchmark: we ran an NGINX process on a container with the NAT or AF_GRAFT and ran the simple GET benchmark using the siege from the other machine. We measured throughput in both bit-per-second and transaction-per-second while changing the file sizes transferred. Note that the files were zero-filled files, and the siege did not use Keep-Alive for persistent connections.

Figure 13a and 13b show the result of the single HTTP session benchmark. In bit-per-second, throughput increases with the file size. Especially when the file size exceeds $16 \mathrm{~K}$ bytes, AF_GRAFT outperforms the NAT. Moreover, it seems that the maximum throughput of NGINX with the NAT for a single HTTP session is approximately $17 \mathrm{Gbps}$. Meanwhile, AF_GRAFT achieves over 25 Gbps with 64M-byte files. For less than $1 \mathrm{M}$-byte small-sized files, AF_GRAFT improves throughput in transaction-per-second as shown in Figure 13b.
The latency reduction demonstrated in the previous experiment affects this result. Naturally, the shorter latency can achieve more transactions per second, especially when the data size is small.

Next, we conducted the same experiment with 50 concurrent sessions. Figure $13 \mathrm{c}$ and $13 \mathrm{~d}$ show the results. The throughput in bit-per-second increased and reached $35 \mathrm{Gbps}$, which is the upper limit of the network stack performance shown in the baseline benchmark, with over $1 \mathrm{M}$ - or $4 \mathrm{M}$ byte files. This upper limit was as expected from the result of throughput measurement with multiple flows. In contrast, Figure 13d shows that the margin between AF_GRAFT and the NAT in transaction-per-second increases compared with the result of the single session benchmark shown in Figure 13b. Until data transfer time becomes dominant overhead due to the large file size (over $64 \mathrm{~K}$ bytes), AF_GRAFT achieves consistently over $25 \mathrm{~K}$ transactions per second, which appears to be the ceiling of the NGINX and its configuration in this experiment. From these results, we conclude that AF_GRAFT can improve the throughput of the containerized HTTP server. In particular, when the size of transferred data is small, transaction-per-second is increased by approximately $10 \%$ (256 bytes) to $40 \%$ ( $64 \mathrm{~K}$ bytes) with 50 concurrent sessions.

\subsection{Message Queue Benchmark}

Next, we measured ZeroMQ throughput and latency as a containerized application. Figure 14 shows the result of throughput measurement. In this experiment, the benchmark application in the container sent messages, and the other machine measured the throughput. As well as the HTTP benchmark, the throughput increases following the increase of the message size. When message size exceeds $64 \mathrm{~K}$ bytes, AF_GRAFT reaches the ceiling on the throughput ( $35 \mathrm{Gbps}$ ). The throughput via the NAT is approximately half of the AF_GRAFT. This result also demonstrates the network performance improvement by AF_GRAFT. Note that, this benchmark application used a single persistent connection to send and receive multiple messages. Therefore, message-per-second achieves the same result as the throughput, unlike the HTTP benchmark where connections were established for every transaction.

Figure 15 shows the result of the latency benchmark on ZeroMQ. In this experiment, the benchmark application in the container sent messages, and the other machine returned them like the latency benchmark using sockperf. The line at the bottom of the key represents the decreasing rate of the latency on each message size. As this line shows, AF_GRAFT decreases the latency, and its rates are over $10 \%$ excluding message sizes of $2-16 \mathrm{~K}$ bytes. With the increase of message size, the time to transfer the message becomes dominant on the round-trip latency. Therefore, the rate increases up to 


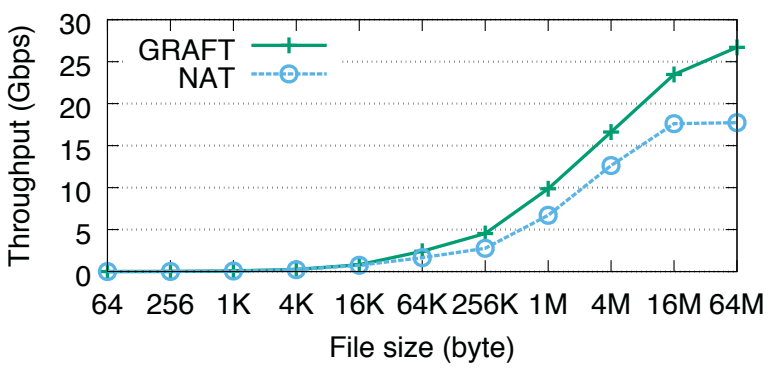

(a) Throughput (Gbps) with one session

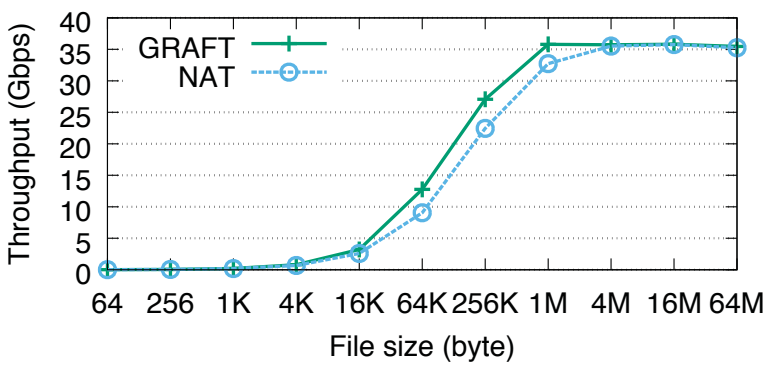

(c) Throughput (Gbps) with 50 concurrent sessions

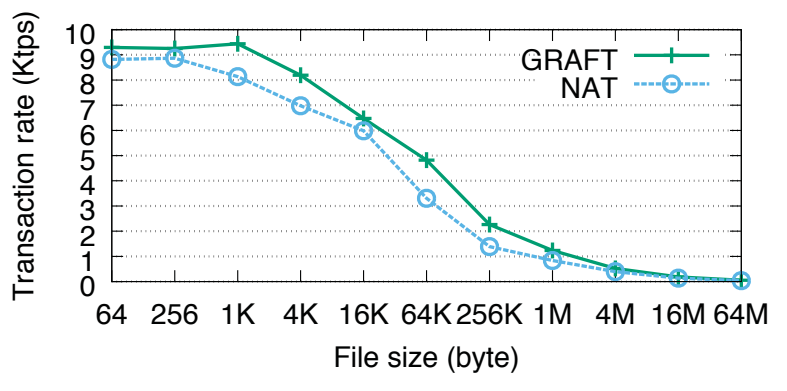

(b) Transaction-per-second with one session

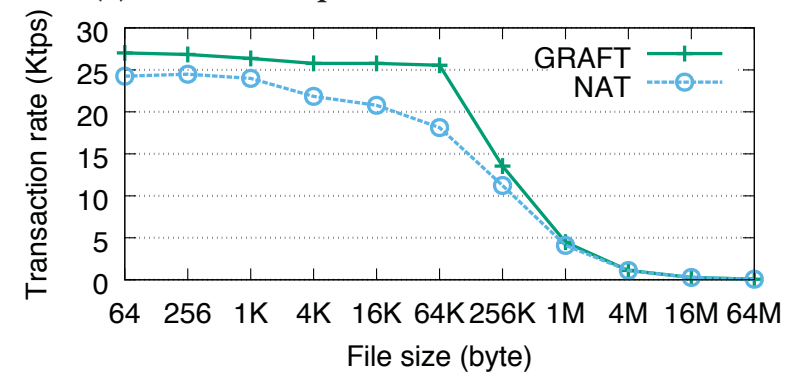

(d) Transaction-per-second with 50 concurrent sessions

Figure 13: HTTP benchmark result.

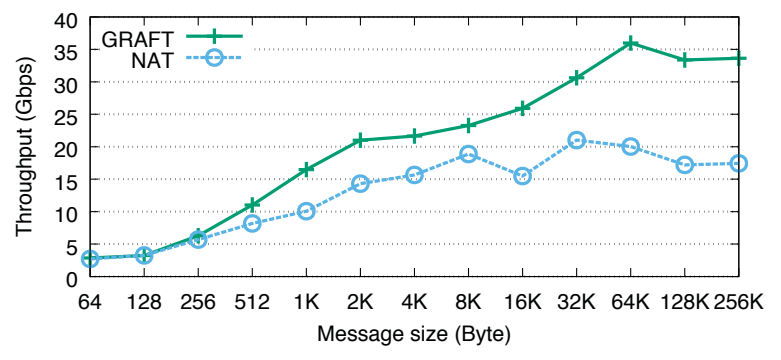

Figure 14: Throughput on the ZeroMQ benchmark.

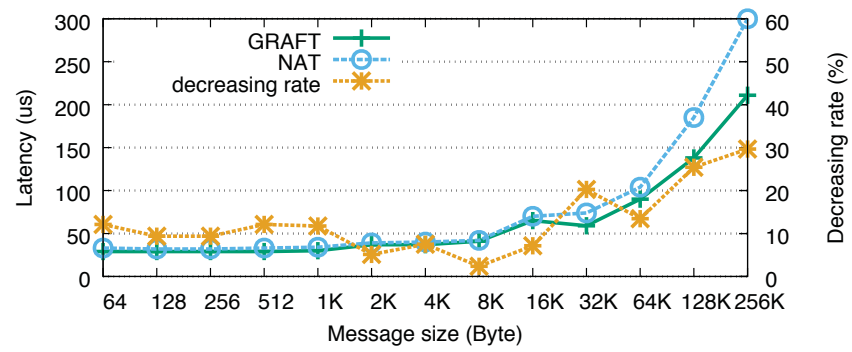

Figure 15: Latency on the ZeroMQ benchmark.

$30 \%$ with $256 \mathrm{~K}$ bytes. These results also demonstrate that AF_GRAFT can contribute to network performance for containerized applications.

\section{RELATED WORK}

FreeFlow [36], which is an optimizing network stack approach, replaces container network stacks and host network stacks with a special network library and an overlay router implementation, respectively. FreeFlow achieved significant throughput and latency improvement by the optimized network stack using RDMA-based shared memory communication. The containers communicate with others in the same host across shared memory space with RDMA API provided by the network library. Moreover, the overlay router in the host space offers inter-host container communication over the RDMA- or DPDK-based data plane. As we noted, socketgrafting does not depend on implementations of network stacks. Therefore, AF_GRAFT could be integrated into the network library and could provide a new data path bypassing the host network stack for greater throughput improvement.

Cilium [5] also attempts to reinvent host network stacks. Instead of DPDK or netmap, Cilium utilizes the extended Berkeley Packet Filter (eBPF) [30] and eXpress Data Path [1]. eBPF, which derives from the Berkeley Packet Filter [23], provides an instruction set for programming various functionalities such as packet handling and function tracing. The Linux kernel has the infrastructure for executing eBPF bytecode inside its kernel. By using eBPF, Cilium implements a data path that can replace the host network stack. The Cilium data path achieves lower overhead than the current packet filter mechanism of Linux (iptables), and it can perform upper-layer firewalls: HTTP, gRPC, and Apache Kafka. 
On the other hand, although the Cilium data path is low overhead, its network stack architecture still involves the two network stacks for containers and routing in the host.

AppSwitch $^{4}$ [33] has a similar mechanism to AF_GRAFT. It decouples applications from underlying networks. Sockets opened in containers are overridden and converted into UNIX domain sockets. The converted sockets are connected to a service router that forwards data from the sockets to the other sockets across the separation between host and container network stacks. As a result, the applications do not need to be aware of the detail of networks, e.g., IP address and port numbers. This decoupling provides manageability and flexibility in modern distributed applications. In contrast to AF_GRAFT that focuses on network performance, AppSwitch focuses on the manageability of containers and their network identifiers by the decoupling. Its UDP throughput between two containers on the same host shown in [33] is less than one-third of that of AF_GRAFT, although the experimental machines are different. The UNIX domain sockets and the service routers may cause overhead in comparison with AF_GRAFT.

Socket-outsourcing [16] proposed a communication channel between the socket layer in virtual machines and the socket layer in host network stacks. The modified socket layer in the virtual machine invokes socket operations on the host network stack using an RPC via a shared memorybased communication channel. Socket-grafting resembles this method. In contrast to socket-outsourcing, which focuses on virtual machines, socket-grafting focuses on containers, so that its communication channel is function call across the separation of network stacks. Moreover, socketoutsourcing does not have a namespace separation mechanism like the graft endpoints and does not provide APIs to specify which socket is outsourced. Therefore, the combination of AF_GRAFT as an API and socket-outsourcing as a communication channel across the virtualization layer may apply to virtual machines.

\section{LIMITATIONS}

Because socket-grafting is a communication channel design in the socket layer, it is not applicable to aspects of other layers such as the performance of TCP/IP protocol processing and low-level packet handling. This section briefly discusses the limitations of socket-grafting.

The network performance improvement by socket-grafting does not outperform the performance of native hosts because it uses the existing kernel network stack implementation. AF_GRAFT is a new communication channel for containers, and it does not improve network stacks themselves. Improving network stacks is still an interesting research topic for

\footnotetext{
${ }^{4}$ http://appswitch.io/
}

both academic and industrial communities [19, 32, 35]. These techniques improve network stack performances, although the network stack architecture of containers-the two network stacks-does not change. When high-speed network stacks become more popular and are deployed in container environments, socket-grafting will still provide a communication channel that is a very low overhead alternative to virtual interfaces and the host network stack performing NAT routers.

Because AF_GRAFT is an enhancement on the socket layer, applications that are sensitive to networks do not work with AF_GRAFT. For example, AF_GRAFT cannot be applied to container-based Network Function Virtualization (NFV) [12, $20,37]$. In container-based NFV, virtual network functions packaged in containers process and deliver packets using low-level packet I/O mechanisms such as virtual interfaces or high-speed packet I/O. They focus on low-level packets and work under the socket layer. Therefore, AF_GRAFT does not help such network-sensitive applications. Applications focusing on only data above the socket layer can derive the benefit of AF_GRAFT.

\section{CONCLUSION}

In this paper, we have proposed a method that grafts sockets in containers onto sockets in host network stacks. This method, socket-grafting, enables the bypassing of container network stacks and virtual interfaces. As a result, it overcomes the network performance degradation due to the container network stack architecture. We developed AF_GRAFT as an actual mechanism of socket-grafting. AF_GRAFT provides the API for grafting sockets in the familiar socket API, and it performs access control through the bind() system call for preventing malicious or unintended uses. In addition, the system call interception technique enables existing applications to coexist with AF_GRAFT without modifications to their codes. Our evaluation demonstrated that AF_GRAFT could achieve the throughput and latency of containers comparable with the native hosts. Also, the other benchmarks showed 10-40\% throughput improvement in the containerized HTTP server, and up to double the throughput and $30 \%$ shorter latency in the ZeroMQ in comparison with the traditional NAT-based container network.

Finally, we plan to integrate AF_GRAFT into network mechanisms of current container platforms: docker and $\mathrm{Ku}-$ bernetes. This integration would provide a convenient way to derive the benefit from socket-grafting in practical containerbased service infrastructures.

\section{ACKNOWLEDGEMENT}

This work was supported by MEXT KAKENHI, Grant Number JP18K18034. 


\section{REFERENCES}

[1] 2016. XDP - IO Visor Project. (2016). https://www.iovisor.org/ technology/xdp

[2] 2017. Intel Data Plane Development Kit. (2017). http://dpdk.org/

[3] 2017. Linux Containers. (2017). https://linuxcontainers.org/

[4] 2018. Apache OpenWhisk is a serverless event-based programming service and an Apache Incubator project. (March 2018). https://github. com/apache/incubator-openwhisk

[5] 2018. Cilium. (2018). https://cilium.io/

[6] 2018. Container Network Interface - networking for Linux containers. (2018). https://github.com/containernetworking/cni

[7] 2018. Kubernetes | Production-Grade Container Orchestration. (2018). https://kubernetes.io/

[8] 2018. networking:iproute2 [Linux Foundation Wiki]. (Feb. 2018). https://wiki.linuxfoundation.org/networking/iproute2

[9] 2018. rkt, a security-minded, standards-based container engine. (2018). https://coreos.com/rkt/

[10] 2018. upa/af-graft. (2018). https://github.com/upa/af-graft

[11] Jake Adriaens. 2017. Google Cloud Platform Blog: Andromeda 2.1 reduces GCP's intra-zone latency by $40 \%$. (Nov. 2017). https://cloudplatform.googleblog.com/2017/11/ Andromeda-2-1-reduces-GCPs-intra-zone-latency-by-40-percent. html

[12] J. Anderson, H. Hu, U. Agarwal, C. Lowery, H. Li, and A. Apon. 2016. Performance considerations of network functions virtualization using containers. In 2016 International Conference on Computing, Networking and Communications (ICNC). 1-7. https://doi.org/10.1109/ICCNC.2016. 7440668

[13] A. Balalaie, A. Heydarnoori, and P. Jamshidi. 2016. Microservices Architecture Enables DevOps: Migration to a Cloud-Native Architecture. IEEE Software 33, 3 (May 2016), 42-52. https://doi.org/10.1109/MS. 2016.64

[14] J. Claassen, R. Koning, and P. Grosso. 2016. Linux containers networking: Performance and scalability of kernel modules. In NOMS 2016 - 2016 IEEE/IFIP Network Operations and Management Symposium. 713-717. https://doi.org/10.1109/NOMS.2016.7502883

[15] Jonathan Corbet. 2007. Large receive offload [LWN.net]. (Aug. 2007) https://lwn.net/Articles/243949/

[16] Hideki Eiraku, Yasushi Shinjo, Calton Pu, Younggyun Koh, and Kazuhiko Kato. 2009. Fast Networking with Socket-outsourcing in Hosted Virtual Machine Environments. In Proceedings of the 2009 ACM Symposium on Applied Computing (SAC '09). ACM, New York, NY, USA, 310-317. https://doi.org/10.1145/1529282.1529350

[17] W. Felter, A. Ferreira, R. Rajamony, and J. Rubio. 2015. An updated performance comparison of virtual machines and Linux containers. In 2015 IEEE International Symposium on Performance Analysis of Systems and Software (ISPASS). 171-172. https://doi.org/10.1109/ISPASS.2015. 7095802

[18] Sean Hefty. 2012. RSOCKETS. (March 2012). http: //downloads.openfabrics.org/Media/Monterey_2012/2012_ Workshop_Mon_Rsockets.pdf

[19] Michio Honda, Felipe Huici, Costin Raiciu, Joao Araujo, and Luigi Rizzo. 2014. Rekindling Network Protocol Innovation with User-level Stacks. SIGCOMM Comput. Commun. Rev. 44, 2 (April 2014), 52-58. https://doi.org/10.1145/2602204.2602212

[20] Yang Hu, Mingcong Song, and Tao Li. 2017. Towards "Full Containerization" in Containerized Network Function Virtualization. In Proceedings of the Twenty-Second International Conference on Architectural Support for Programming Languages and Operating Systems (ASPLOS '17). ACM, New York, NY, USA, 467-481. https://doi.org/10.1145/3037697.3037713

[21] Junichiro Itoh. 2003. Implementing AF-independent application. (May 2003). http://www.kame.net/newsletter/19980604/
[22] James Lewis and Martin Fowler. 2014. Microservices. (March 2014). https://martinfowler.com/articles/microservices.html

[23] Steven McCanne and Van Jacobson. 1993. The BSD Packet Filter: A New Architecture for User-level Packet Capture. In Proceedings of the USENIX Winter 1993 Conference Proceedings on USENIX Winter 1993 Conference Proceedings (USENIX'93). USENIX Association, Berkeley, CA, USA, 2-2. http://dl.acm.org/citation.cfm?id=1267303.1267305

[24] Dirk Merkel. 2014. Docker: Lightweight Linux Containers for Consistent Development and Deployment. Linux fournal. 2014, 239, Article 2 (March 2014). http://dl.acm.org/citation.cfm?id=2600239.2600241

[25] R. Morabito, J. KjÃdllman, and M. Komu. 2015. Hypervisors vs. Lightweight Virtualization: A Performance Comparison. In 2015 IEEE International Conference on Cloud Engineering. 386-393. https://doi.org/10. 1109/IC2E.2015.74

[26] Cristina Opriceana and Hajime Tazaki. 2017. Network stack personality in Android phone. (2017). https://www.netdevconf.org/2.2/papers/ tazaki-mptcp-talk.pdf

[27] Simon Peter, Jialin Li, Irene Zhang, Dan R. K. Ports, Doug Woos, Arvind Krishnamurthy, Thomas Anderson, and Timothy Roscoe. 2014. Arrakis: The Operating System is the Control Plane. In 11th USENIX Symposium on Operating Systems Design and Implementation (OSDI 14). USENIX Association, CO, 1-16. https://www.usenix.org/conference/osdi14/ technical-sessions/presentation/peter

[28] Kevin Pulo. 2009. Fun with LD_PRELOAD. (Jan. 2009). https://linux. org.au/conf/2009/slides/172.pdf

[29] Luigi Rizzo. 2012. netmap: A Novel Framework for Fast Packet I/O. In 2012 USENIX Annual Technical Conference (USENIX ATC 12). USENIX Association, Boston, MA, 101-112. https://www.usenix.org/ conference/atc12/technical-sessions/presentation/rizzo

[30] Jay Schulist, Daniel Borkmann, and Alexei Starovoitov. 2018. Linux Socket Filtering aka Berkeley Packet Filter (BPF). (Jan. 2018). https: //www.kernel.org/doc/Documentation/networking/filter.txt

[31] Amazon Web Services. 2018. AWS Lambda - Serverless Compute. (2018). https://aws.amazon.com/lambda/?nc1=h_ls

[32] Solarflare. 2013. OpenOnload. (2013). http://www.openonload.org/

[33] Dinesh Subhraveti, Sri Goli, Serge Hallyn, Ravi Chamarthy, and Christos Kozyrakis. 2017. AppSwitch: Resolving the Application Identity Crisis. (2017). arXiv:arXiv:1711.02294

[34] Mellanox Technologies. 2018. Mellanox/sockperf: Network Benchmarking Utility. (March 2018). https://github.com/Mellanox/sockperf

[35] Kenichi Yasukata, Michio Honda, Douglas Santry, and Lars Eggert. 2016. StackMap: Low-Latency Networking with the OS Stack and Dedicated NICs. In 2016 USENIX Annual Technical Conference (USENIX ATC 16). USENIX Association, Denver, CO. https://www.usenix.org/conference/atc16/technical-sessions/ presentation/stackmap-low-latency-networking-os-stack-and

[36] Tianlong Yu, Shadi Abdollahian Noghabi, Shachar Raindel, Hongqiang Liu, Jitu Padhye, and Vyas Sekar. 2016. FreeFlow: High Performance Container Networking. In Proceedings of the 15th ACM Workshop on Hot Topics in Networks (HotNets '16). ACM, New York, NY, USA, 43-49. https://doi.org/10.1145/3005745.3005756

[37] Wei Zhang, Guyue Liu, Wenhui Zhang, Neel Shah, Phillip Lopreiato, Gregoire Todeschi, K.K. Ramakrishnan, and Timothy Wood. 2016. OpenNetVM: A Platform for High Performance Network Service Chains. In Proceedings of the 2016 Workshop on Hot Topics in Middleboxes and Network Function Virtualization (HotMIddlebox '16). ACM, New York, NY, USA, 26-31. https://doi.org/2940147.2940155

[38] Yang Zhao, Nai Xia, Chen Tian, Bo Li, Yizhou Tang, Yi Wang, Gong Zhang, Rui Li, and Alex X. Liu. 2017. Performance of Container Networking Technologies. In Proceedings of the Workshop on Hot Topics in Container Networking and Networked Systems (HotConNet '17). ACM, New York, NY, USA, 1-6. https://doi.org/10.1145/3094405.3094406 